\title{
COMPORTAMENTO DA CULTIVAR DE MACIEIRA FUJI (Malus domestica, Borkh.) SOBRE TRES DIFERENTES PORTA-ENXERTOS ${ }^{1}$
}

\author{
FREDERICO DENARDI ${ }^{2} \&$ MÁRCIA MONDARDO SPENGLER ${ }^{3}$
}

\begin{abstract}
RESUMO - Verifica-se, a nível mundial, uma forte tendência para o plantio da macieira em alta densidade de cultivo. Neste sistema de plantio, são utilizados porta-enxertos de pequeno porte, conhecidos como anões. O mais utilizado é o M-9, em virtude do forte controle sobre o porte da copa, da precocidade de produção, da alta produtividade e da boa qualidade dos frutos que induz à copa. No Sul do Brasil, por questões de tradição internacional, facilidade de obtenção e do menor custo de investimento no plantio, até recentemente, têm sido plantados porta-enxertos de vigor médio, como o MM-106, o M-7 e o MM-111, para plantios de média densidade. O primeiro é atualmente pouco usado devido à alta suscetibilidade à podridão-do-colo (Phytophthora cactorum). O objetivo deste trabalho foi comparar o desempenho do anão M-9, do semi-anão M-7 e do semivigoroso MM-111 no controle do vigor da copa, na precocidade de produção, na produtividade e no tamanho dos frutos da cv. de macieira Fuji. O experimento foi conduzido em blocos ao acaso, com 4 repetições de 3 plantas por parcela. Como copa, foi utilizada a cv. Fuji, polinizada pela cv. Gala. O experimento foi implantado em 1996, em Fraiburgo-SC, principal pólo produtor de maçãs do País. O espaçamento de cultivo foi de 2,0 m por 5,0 m. O experimento foi conduzido por 4 anos, avaliando-se a precocidade $\left(\mathrm{n}^{0}\right.$ de gemas de flor $/ \mathrm{cm}^{2}$ de área transversal do caule), produção (kg/planta), produtividade ( $\mathrm{t} / \mathrm{ha})$, peso médio dos frutos $(\mathrm{g})$ e distribuição dos frutos por categoria de tamanho (\%). Os resultados obtidos indicaram que o M-9 foi o mais precoce, produzindo, no terceiro ano, 1,94 vez mais gemas de flor que o M-7 e 2,70 vezes mais que o MM-111. Em termos de produção, no terceiro ano, o M-9 produziu 2,53 vezes mais que o M-7 e 2,70 vezes mais que o MM-111. No quarto ano, o M-9 produziu 1,28 vez mais que o M-7 e 1,26 vez mais que o MM-111. O peso médio dos frutos foi de 159,2 g, 135,5 g e 131,2 g, para o M-9, o M-7 e o MM-111, respectivamente. Em termos de distribuição por categoria de tamanho, o M-9 produziu 90,8\% de frutos maiores que $62 \mathrm{~mm}$, o M-7 produziu 79,5\% e o MM-111, 70,9\%, indicando que o M-9, além de mais precoce e mais produtivo, também produz frutos de maior calibre.
\end{abstract}

Termos para indexação: Vigor, precocidade, produtividade, tamanho de frutos

\section{“BEHAVIOUR OF THE APPLE CULTIVAR FUJI (Malus domestica, Borkh.) ONTO THREE DIFFERENT ROOTSTOCKS"}

\begin{abstract}
There is a worldwide tendency for planting apples at high density system onto the so called dwarf apple rootstocks, which have a strong effect on controlling the vigor of the trees. The most used is the M-9 due to its efficiency on vigor control, precocity of flowering, high productivity and also good fruit quality it induces to the scion varieties. In South of Brazil more vigorous rootstocks, as MM-106, M-7 and MM-111, have been widely used because worldwide tradition, lower initial cost as they require less trees/ha, and their facility of propagation The objective of this work was to compare the dwarf M-9 with the semi-dwarf M-7 and the semi-vigorous MM-111 concerned to vigor control, precocity of flowering, productivity and fruit quality of the apple cultivar Fuji. The statistical design was on completely randomized blocks, with 4 replications of three trees on each plot. The main cultivar was Fuji, pollinated by cv. Gala. The experiment was established in 1996 in Fraiburgo, the main apple producer area in the country. The layout of planting was 2,0 m within row by $5,0 \mathrm{~m}$ between rows. The study was carried out for four years. The parameters evaluated were precocity ( $\mathrm{n}^{0}$ of flower buds per $\mathrm{cm}^{2}$ of trunk cross sectional area), production $(\mathrm{kg} /$ tree), productivity $(\mathrm{t} / \mathrm{ha})$, fruit weigh $(\mathrm{g})$ and distribution of fruit size (\%). The results obtained on the $3^{\text {rd }}$ season showed that M-9 was the most precocious, producing 1.94 times more flower buds per $\mathrm{cm}^{2}$ of trunk cross sectional area than on M-7 and 2.7 times more than on MM-111. Concerned to production on $3^{\text {rd }}$ season, M-9 produced 2.5 times more than M-7 and 2.7 times more than MM-111. On the $4^{\text {th }}$ season M- 9 produced 1.28 times more than M-7 and 1.26 times more than MM-111 (Table 2). The fruit weight was 159,2 g, 135,5 g and 131,2 g for M-9, M-7 and MM-111, respectively. In terms of distribution of fruit weight, M-9 produced $90,8 \%$ of fruits In the class $>62 \mathrm{~mm}$, against 79,5\% on M-7 and 70,9\% on MM-111, showing that M-9 is not only the most precocious, but it is also the most productive and produces fruits of larger size than M-7 and MM-111.
\end{abstract}

Index terms: Vigor, precocity, productivity, fruit size

1 (Trabalho 154/2000). Recebido: 24/07/2000. Aceito para publicação: 16/10/2001. Apoio financeiro: Associação Brasileira dos Produtores de Maçã - ABPM

2 Pesquisador da Epagri-SC, C.Postal, 591, CEP 89500-000, e-mail denardi@epagri-rct-sc.br, Caçador, SC

3 Pesquisadora da Epagri-SC, C.Postal, 591, CEP 89500-000, e-mail mmondardo@epagri-rct-sc.br, Caçador, SC

Rev. Bras. Frutic., Jaboticabal - SP, v. 23, n. 3, p. 630-633, dezembro 2001 


\section{INTRODUÇÃO}

A maçã é um dos principais produtos agrícolas de Santa Catarina (Hentschke, 1993). O Estado colheu mais de 50\% da produção nacional de 720 mil toneladas em 1999 (Boneti et al., 1999). Entretanto, os produtores ainda se defrontam com alguns problemas, dentre os quais a demora das plantas em iniciar a produção comercial, a baixa qualidade dos frutos, especialmente em tamanho, e o excesso de vigor das plantas.

Verifica-se, a nível mundial, uma forte tendência para o plantio de macieira em alta densidade de cultivo, usando-se portaenxertos anões (Sansavini et al. 1998). As principais justificativas para esta nova tendência são a facilidade de manejo de plantas de pequeno porte sobre este tipo de porta-enxerto (Wertheim, 1998), a alta precocidade em iniciar a frutificação e a eficiência produtiva (Tukey, 1970). A mão-de-obra, em atividades como poda, raleio dos frutos e colheita, representa um importante fator que onera mais os custos de produção em plantas de grande porte.

No sistema de alta densidade, são utilizados portaenxertos de pequeno porte, conhecidos como anões. $\mathrm{O}$ mais utilizado é o M-9, em virtude do forte controle sobre o porte da copa, da precocidade de produção, da alta produtividade e da boa qualidade dos frutos que induz à copa.

No Sul do Brasil, por questões de tradição internacional, facilidade de obtenção e do menor custo de investimento no plantio, têm sido empregados, basicamente, porta-enxertos de vigor médio, dentre os quais se destacam o MM-106, o M-7 e o MM-111. O primeiro atualmente pouco usado devido à alta suscetibilidade à podridão-do-colo (Phythophtora cactorum, Lebert.-Cohn, Schrot). Estes porta-enxertos, que já foram muito utilizados no Hemisfério Norte, produzem copas com porte alto, necessitando de escadas e/ou plataformas móveis para realizar os tratos culturais. A utilização de tais equipamentos reduz a eficiência da mão-de-obra, encarecendo a produção.

Para atender a um mercado consumidor cada vez mais exigente em qualidade, os fruticultores têm buscado portaenxertos mais eficientes no que se refere ao tamanho e à coloração dos frutos. Por outro lado, a capacidade de induzir precocidade em iniciar a produção e alta produtividade são características essenciais para um porta-enxerto, as quais permitem mais rápido retorno dos investimentos de plantio. Pesquisas têm mostrado que, em macieira, em geral, quanto mais anão, mais eficiente temse revelado o porta-enxerto (Tukey, 1970). Por esta razão, os principais países produtores de maçã na Europa, e mais recentemente nos Estados Unidos e Canadá, utilizam principalmente o M-9 e seus diferentes subclones, considerados os mais eficientes dentre os porta-enxertos das séries ' $M$ ' e 'MM' nas características acima (Barritt, 1992).

No Sul do Brasil, os porta-enxertos mais anões, como o M-9 e o M-26, foram pouco utilizados até meados da década de 1990, por exigirem mais tecnologia de manejo das plantas e do solo, e por necessitarem tutoramento, fatores que encarecem o custo inicial de plantio do pomar. No entanto, observa-se atualmente uma tendência de reversão deste processo. Pomares sobre porta-enxertos anões, plantados em alta densidade, estão paulatinamente substituindo os porta-enxertos mais vigorosos.

O objetivo deste estudo foi comparar o desempenho agronômico nas condições edafoclimáticas do Sul do Brasil, de três dos porta-enxertos de diferentes vigores mais utilizados comercialmente, o anão M-9, o semi-anão M-7 e o semivigoroso MM-111.

\section{MATERIAL E MÉTODOS}

O presente trabalho foi extraído de um experimento composto por 17 diferentes porta-enxertos de macieira. Foram excluídos deste trabalho 14 deles, em virtude de se tratarem de seleções, sobre as quais ainda não se pode emitir qualquer recomendação de plantio, enquanto não forem lançadas como novos porta-enxertos. A análise estatística, cujos resultados são aqui referidos, foi aplicada sobre os resultados obtidos com os 17 novos porta-enxertos.

O experimento foi desenvolvido no município de Fraiburgo, em Santa Catarina, principal pólo produtor de maçãs do País, em solo tipo Vacaria, categoria Latossolo, Bruno, Úmico, Distrófico. O relevo é ondulado e a pluvio- metria é de $1660 \mathrm{~mm}$ anuais. O pomar foi implantado no inverno de 1996, obedecendo ao delineamento experimental de blocos ao acaso, com 4 repetições de 3 plantas por parcela. A cultivar copa foi a Fuji e a polinizadora foi a Gala, principais cultivares plantadas no Sul do Brasil. O espaçamento de cultivo foi de $2,0 \mathrm{~m}$ na fila por $5,0 \mathrm{~m}$ entre filas, correspondendo a 1000 plantas por hectare. Os tratamentos (porta-enxertos) estudados foram o M-9 (anão), o M-7 (semi-anão) e o MM-111 (semivigoroso). Os tratamentos fertilizantes e fitossanitários, como também os tratos culturais, seguiram as orientações do Sistema de Produção para a Macieira (Embrater/Embrapa, 1984). Foram avaliadas, anualmente, as seguintes variáveis: a) precocidade na produção comercial (gemas de flor/ $\mathrm{cm}^{2}$ de área transversal do caule); b) produção (kg/planta); c) produtividade acumulada (t/ha); d) peso médio dos frutos $(\mathrm{g})$; e) distribuição dos frutos por categoria de tamanho; e f) porte das plantas (m). A precocidade, dada pelo número de gemas de flor por $\mathrm{cm}^{2}$ de área transversal do caule, medida a $5 \mathrm{~cm}$ acima do ponto de enxertia, expressa a capacidade do porta-enxerto em induzir precocemente a diferenciação de gemas de flor. O tamanho dos frutos é expresso pelo seu peso médio. Na distribuição por tamanho dos frutos, consideraram-se duas categorias: a) frutos com diâmetro menor ou igual a $62 \mathrm{~mm}$; b) frutos com diâmetro maior que $62 \mathrm{~mm}$. O porte foi estabelecido pela altura das plantas, medida no quarto ano após o plantio.

\section{RESULTADOS E DISCUSSÃO}

a) Altura das plantas e precocidade de produção:

Na Tabela 1, é mostrada a altura das plantas e o número de gemas de flor por planta e por $\mathrm{cm}^{2}$ de área transversal do caule - ATC, em ramos de 2 anos.

As plantas da cv. Fuji sobre o M-9 apresentaram porte médio $26,4 \%$ menor que sobre o M-7, porém produziram $1,37 \mathrm{vez}$ mais gemas de flor por planta. A área transversal do caule (ATC) foi 1,41 vez menor sobre o M-9 do que sobre o M-7 e 2,28 vezes menor do que sobre o MM-111. Segundo Barritt (1992), o M-9 e o M-7 têm, respectivamente, $30 \%$ e $60 \%$ do vigor do pé-franco. Carlson (1971) relata que, no espaçamento adequado para cada tipo de porta-enxerto, quanto mais anão for este, maior será a 
produção acumulada da copa enxertada sobre ele.

$\mathrm{O}$ número de gemas de flor por $\mathrm{cm}^{2}$ de ATC, o qual expressa a precocidade do porta-enxerto, foi 1,93 vez maior no M-9 que no M-7 e 2,71 vezes maior no M-9 que no MM-111, indicando que o M-9 é efetivamente bem mais precoce que o M7 e o MM-111. Westwood et al. (1986), em estudos de produção acumulada e eficiência produtiva de uma série de porta-enxertos, realizados do $2^{0}$ ao $4^{0}$ anos após o plantio, observaram que o M9, nestas três safras, produziu 33,4 kg/planta, enquanto o M-7 produziu apenas $17,6 \mathrm{~kg} /$ planta e o MM-111 24,9 kg/planta. Estes autores observaram que a eficiência produtiva do M-9 foi de $2,31 \mathrm{~kg} / \mathrm{cm}^{2}$ de ATC, a do M-7 foi de $0,95 \mathrm{~kg}$ e a do MM-111 foi de $1,10 \mathrm{~kg}$.

A área de produção do M-9 raramente chega aos 3 metros de altura, permitindo a realização dos tratos culturais e de colheita, sem necessidade de escadas. Devido à sua alta precocidade de produção, requer cuidados especiais no raleio de frutos. Muitas vezes, além do raleio químico, necessita de um repasse manual para a complementação do raleio. Apresenta, como principais limitações, a fragilidade do sistema radicular e do lenho, requerendo, por isso, tutoramento das plantas; e a alta suscetibilidade ao pulgão-lanígero (Eriosoma lanigerum, Hausm.), praga importante no Sul do Brasil e que ataca, principalmente, o sistema radicular.

O M-7 e o MM-111, por outro lado, são porta-enxertos mais vigorosos, cultivados em média e baixa densidades de cultivo, respectivamente. Como principais limitações, o MM-111 é lento em iniciar a frutificação, e o M-7 é também altamente suscetível ao pulgão-lanígero e ao rebrotamento ao nível do colo das plantas.

b) Produção por planta e produtividade por hectare

Os resultados da Tabela 2 evidenciam a influência da precocidade de produção e da produtividade do M-9, especialmente no $3^{0}$ ano após o plantio. O M-9, bem mais precoce que os demais, também atinge a plena produção mais rapidamente. No $3^{0}$ ano, enquanto o M-7 e o MM-111 não produziram $3 \mathrm{~kg}$ de frutos por planta, o M-9 produziu, aproximadamente, $7 \mathrm{~kg} /$ planta.

No quarto ano, ficou evidenciado que as diferenças de produção entre os três porta-enxertos foram bem menores que as obtidas no terceiro ano. Isso mostra que as diferenças em produção vão diminuindo à medida que as plantas atingem a idade de produção. No entanto, a precocidade em iniciar a produção do M-9 contribui para um melhor rendimento do pomar, principalmente nos primeiros anos após o plantio. A produtividade acumulada até o $4^{0}$ ano foi quase $10 \mathrm{t} /$ ha maior neste porta-enxerto que no M-7 e no MM-111 (Tab. 2). Barritt (1992) relata que a cv. Starkspur Supreme Delicious sobre o M-9 produziu, até o $10^{\circ}$ ano, 326,32 t/ha, contra 219,53 t/ha sobre o M7.

O espaçamento utilizado foi adequado até o $4^{0}$ ano para os porta-enxertos M-7 e MM-111, mas muito amplo para o M-9. Usando-se espaçamento mais denso para este último, poder-seia obter produção comercial mais cedo e produtividade maior do que a apresentada na Tabela 2.

c) Peso médio e distribuição por categoria de tamanho dos frutos O raleio de frutos em macieira é uma prática de rotina. Esta prática visa a melhorar o calibre dos frutos e, ao mesmo tempo, evitar a alternância de produção.

No terceiro ano após o plantio, procurou-se fazer o raleio, mantendo-se igual densidade de frutos nos cachos florais, via de regra, um ou, no máximo, dois frutos por cacho em todos os tratamentos.

Segundo Tukey (1970), porta-enxertos mais vigorosos como M-7 e o MM-111 levam mais tempo para atingirem a idade de produção comercial. Este fato foi observado neste experimento. Por causa do pequeno número de cachos florais nestes dois porta-enxertos por ocasião do raleio, a densidade de frutos, após efetuado o raleio, foi muito baixa, em relação ao que ficou no M9. Mesmo com uma produção 2,7 vezes maior que a do MM-111, o M-9 produziu frutos significativamente maiores, com quase $160 \mathrm{~g}$, contra 131,5 g no MM-111 (Tab. 3). Isto mostra que a produção de frutos grandes é uma característica varietal deste porta-enxerto. A distribuição dos frutos por categoria de tamanho confirma isto. Mais de $90 \%$ deles apresentaram tamanho acima de $62 \mathrm{~mm}$ de diâmetro, contra 70,9\% no MM-111. O M-9 é um porta-enxerto que tem também a vantagem de ser mais resistente à podridão-do-colo que o M-7 e o MM-111, e de produzir frutos mais coloridos, devido à copa mais compacta, com ramos curtos, expondo mais os frutos ao sol. No entanto, requer solos de alta fertilidade, principalmente em matéria orgânica, com boa retenção de umidade, mas ao mesmo tempo bem drenados. Em virtude de produzir plantas de pequeno porte e de ser altamente produtivo, em condições de deficiência hídrica e/ou nutricional, poderá entrar precocemente em declínio vegetativo. Este processo de declínio vegetativo é muito mais difícil de ser revertido em porta-enxertos anões, como o M-9, do que em porta-enxertos mais vigorosos, como o MM-111.

Uma das vantagens mais relevantes do M-9 é a rápida entrada em produção comercial, permitindo mais rápido retorno do capital inicial investido no plantio do pomar. Via de regra,

TABELA 1 - Altura das plantas, área transversal do caule e número de gemas floríferas obtido no $3^{0}$ ano após o plantio da cultivar de macieira Fuji enxertada sobre os porta-enxertos M-9, M-7 e MM-111 - Fraiburgo-SC - safra 1999/2000

\begin{tabular}{c|c|c|c|c}
\hline PORTA- & ALTURA DAS & AREA TRANSVERSAL & \multicolumn{2}{|c}{ GEMAS DEFLOPOR } \\
\cline { 4 - 6 } ENXERTO & PLANTAS $(\mathrm{m})$ & DO CAULE - ATC $\left(\mathrm{cm}^{2}\right)^{1 /}$ & planta & $\mathrm{cm}^{2}$ ATC C $^{2 /}$ \\
\hline M-9 & 2,61 & 8,92 & 37,6 & $4,22 \mathrm{a}$ \\
M-7 & 3,30 & 12,57 & 27,4 & $2,18 \mathrm{~b}$ \\
M M-111 & 3,85 & 20,35 & 31,7 & $1,56 \mathrm{~b}$ \\
\hline
\end{tabular}

\footnotetext{
${ }^{1 /}$ Medida tomada a $5 \mathrm{~cm}$ acima do ponto de enxertia

${ }^{2 /}$ Valores seguidos pela mesma letra não diferem entre si, pelo teste de Duncan, a 5\% de propabilidade.
} 
TABELA 2 - Produção por planta e produtividade acumulada da cultivar de macieira Fuji enxertada sobre os porta-enxertos M-9, M7 e MM-111 - Fraiburgo-SC - safra 1999/2000

\begin{tabular}{|c|c|c|c|c|}
\hline \multirow{2}{*}{$\begin{array}{l}\text { PORTA- } \\
\text { ENXERTO }\end{array}$} & \multirow{2}{*}{$\begin{array}{l}\text { PLANTAS/ } \\
\text { HECTARE }\end{array}$} & \multicolumn{2}{|c|}{ PRODUÇÃ O (kg/planta) } & \multirow{2}{*}{$\begin{array}{l}\text { PRODUTIVIDADE } \\
\text { ACUMULADA }(\mathrm{t} / \mathrm{ha})\end{array}$} \\
\hline & & safra $98 / 99^{2 /}$ & safra $99 / 00^{2 /}$ & \\
\hline M -9 & 1.000 & 6,94 a & 25,73 a & 32,67 \\
\hline M - 7 & 1.000 & $2,74 b$ & $20,11 \quad b$ & 22,85 \\
\hline M M - 111 & 1.000 & $2,57 \mathrm{~b}$ & $20,48 \quad b$ & 23,05 \\
\hline
\end{tabular}

1/ Dados referentes ao $3^{0}$ e ao $4^{0}$ anos após o plantio, respectivamente

${ }^{2 /}$ Valores seguidos pela mesma letra não diferem estatisticamente entre si, pelo teste de Duncan, a 5\% de probabilidade.

TABELA 3 - Peso médio e distribuição dos frutos por categoria de tamanho da cultivar de macieira Fuji enxertada sobre os portaenxertos M-9, M-7 e MM-111 - $3^{0}$ ano após o plantio - safra 98/99 - Fraiburgo-SC

\begin{tabular}{|c|c|c|c|}
\hline \multirow{2}{*}{$\begin{array}{c}\text { PORTA- } \\
\text { ENXERTO }\end{array}$} & \multirow{2}{*}{$\begin{array}{l}\text { PESO MÉDIO } \\
\text { FRUTOS }(\mathrm{g})\end{array}$} & \multicolumn{2}{|c|}{ D IS T R IB U IÇ Ã O P O R T A M A N H O (\%) } \\
\hline & & $? 62 \mathrm{~m} \mathrm{~m}$ & $>62 \mathrm{~m} \mathrm{~m}$ \\
\hline $\mathrm{M}-9$ & 159,2 a & 9,2 & 90,8 \\
\hline $\mathrm{M}-7$ & $135,5 \mathrm{~b}$ & 20,5 & 79,5 \\
\hline M M - 111 & $131,2 \quad b$ & 29,1 & 70,9 \\
\hline
\end{tabular}

${ }^{1 /}$ Valores seguidos pela mesma letra não diferem estatisticamente entre si, pelo teste

de Duncan, ao nível de 5\% de probabilidade.

estes custos iniciais são mais elevados nos porta-enxertos anões, devido ao tutoramento e ao maior número de mudas necessárias por hectare. Por todas estas razões, o M-9 é um porta-enxerto de pequeno porte, ideal para plantios em alta densidade.

\section{CONCLUSÕES}

Os resultados obtidos neste estudo permitiram as seguintes conclusões:

1. O porta-enxerto de macieira M-9 induz maior precocidade para iniciar a produção na cv. Fuji que o M-7 e o MM-111, iniciando a produção comercial já no terceiro ano após o plantio do pomar, no espaçamento de $2,0 \mathrm{~m} \times 5,0 \mathrm{~m}$.

2. Na cv. Fuji, o porta-enxerto M-9 induz maior produção por planta do que os porta-enxertos M-7 e o MM-111, até o quarto ano após o plantio.

3. No espaçamento de 2,0 m x 5,0 m, a cv. Fuji sobre o M9 é mais produtiva que sobre os porta-enxertos M-7 e o MM-111 até o $4^{0}$ ano.

4. A cv. Fuji sobre o M-9, além de mais produtiva nos primeiros 4 anos, produz frutos de maior calibre que sobre o M7 ou sobre o MM-111.

\section{REFERÊNCIAS BIBLIOGRÁFICAS}

BARRITT, B.H. Intensive Orchard Management. Yakima: Good Fruit Grower, 1992.212p.

BONETI, J.I.S.; CESA, J.D.; PETRI, J.L.; HENTSCHKI, R. Cadeias produtivas do Estado de Santa Catarina: maçã. Florianópolis:
Epagri, 1999.94p. (Boletim Técnico, 105).

CARLSON, R.F. Fruit trees-dwarfing and propagation: Horticultural Report. . Michigan: Michigan State University, $1971.72 \mathrm{p}$.

EMPRESA BRASILEIRA DE ASSISTÊNCIA TÉCNICA E EXTENSÃO RUAL/EMPRESA BRASILEIRA DE PESQUISA AGROPECUÁRIA. Sistemas de produção para a maçã. 2. ed. rev. Florianópolis: EMPASC/ACARESC, 1984. 100 p. (Boletim, $380)$.

HENTSCHKE, R. Maçã: estudo da situação catarinense frente ao MERCOSUL. Florianópolis: EPAGRI, 1993. 70 p. (Documentos, 148).

SANSAVINI, S.; ERRANI, A. Fruticoltura ad Alta Densità: Impianti, Forme D'alevamento e Tecniche di potatura. Edagricole, Bologna. 593 p. 1998.

TUKEY, H. Dwarfed Fruits Trees. 2. ed. Londres: The MacMillan Campany, 1970.

WERTHEIM, S.J. Rootstock Guide: Apple, Pear, Cherry, European Plum. Wilhelminadorp: Fruit Research Station, 1998. 144p.

WESTWOOD, M.N.; LOMBARD, P.B.; ROBBINS, S. ; BJORNSTAD, H.O. Tree size and performance of young apple trees on nine cultivars on several growth-controlling rootstocks. HortScience, Alexandria, v.21, n.6, p. 1365-1366, 1986. 